\title{
Perforación esofágica. Experiencia clínica y actualización del tema
}

\author{
Italo Braghetto M, Alberto Rodríguez N, Attila Csendes J, \\ 0 wen Korn B.
}

\section{An update on esophageal perforation}

\begin{abstract}
Esophageal perforation is a complicated clinical entity that demands a high level of diagnostic and therapeutic skills. The management alternatives vary from conservative treatment to esophagectomy, including primary suture and esophageal exclusion. This paper is a review of the literature and personal experience with this condition, focusing on etiology, clinical presentation, diagnostic workout, treatment, complications and mortality. Finally, we propose a management algorithm (Rev Méd Chile 2005; 133: 1233-41).
\end{abstract}

(Key Words: Digestive system; Esophagoscopy; Esophageal perforation.

Recibido el 17 de agosto, 2004. Aceptado el 28 de julio, 2005.

Departamento de Cirugía, Hospital Clínico Universidad de Chile.

A ún en nuestros días, la perforación esofágica continúa siendo un gran desafío clínico, ya que exige una alta sospecha diagnóstica, un juicio clínico excelente y un manejo adecuado, basado en múltiples procedimientos para un órgano de limitada plasticidad. Primero hay que señalar que es una verdadera emergencia, ya que es una enfermedad grave con alta morbi-mortalidad ${ }^{1-4}$. El tratamiento tardío aumenta la magnitud de la contaminación e inflamación, reduciendo así la posibilidad de un cierre efectivo de la perforación, desarrollándose como evento final, una falla multiorgánica debida a sepsis no controlada ${ }^{3,5}$. Como segundo punto, esta patología requiere una gran acuciosidad diagnóstica, basada en una alta sospecha clínica, ya que es poco frecuente y tiene una

Correspondencia a: Dr. Italo Braghetto M. Hospital Clínico Universidad de Chile. Santos Dumont 999, Independencia. Fax: 735-02-51. E mail: ibraghet@redclinica.uchile.cl gran variedad de presentaciones, que abarca desde un cuadro típico a algunos cuadros inespecíficos y con poca signología. Por último, la decisión más crítica consiste en la elección del manejo adecuado para cada caso, siendo este el punto más controversial, ya que hay que optar por un tratamiento no invasivo versus uno instrumental, que comprende desde la instalación de una endoprótesis hasta la esofagectomía, incluyendo la sutura primaria de la lesión (con o sin refuerzo) o la desfuncionalización esofágica para un cierre en un segundo tiempo ${ }^{7}$. Para decidir el tratamiento óptimo algunos de los parámetros que tienen que ser evaluados son el estado general del paciente, el tiempo de evolución, la localización de la perforación y la presencia de patologías esofágicas previas, entre otras.

El objetivo del presente trabajo es realizar una revisión de la etiología, la clínica (orientada básicamente en cuándo sospechar la patología), los métodos diagnósticos existentes y delinear las pautas para orientar la toma de decisiones en el tratamiento a seguir, proponiendo un algoritmo de tratamiento. 


\section{ETIOLOGíA}

La causa más común de perforación esofágica hoy es la perforación iatrogénica, secundaria a la instrumentalización endoscópica del esófago. Las otras etiologías son: rotura espontánea, traumatismo secundario a la ingestión de cuerpo extraño y otras poco frecuentes (tumores, ingestión de cáusticos, lesión por medicamentos retenidos, esofagitis severa, intubación endotraqueal difícil, tiroidectomía). La Tabla 1 muestra la distribución de etiologías según estudios realizados ${ }^{4,7-17}$.

Instrumental. La endoscopia diagnóstica y terapéutica es una parte integral de cualquier centro médico importante de la actualidad y al ser un procedimiento invasivo, tiene riesgo de complicaciones. Con la antigua esofagoscopia rígida existía un riesgo de 0,1 a $0,4 \%$ de perforación. El riesgo de perforación asociada a la endoscopia flexible diagnóstica es muy baja y en la literatura tiene un rango entre $0,006 \%$ y $0,06 \% 18$. El riesgo aumenta considerablemente con el uso de intervenciones terapéuticas. La incidencia de perforación con balón hidrostático ha sido estimada en 0,3\%. La dilatación en acalasia acarrea un mayor riesgo, que ha sido estimado en 2 a $6 \% 6,20$. También es alto el riesgo en ciertas situaciones clínicas como estenosis por irradiación y malignas, teniendo éstas un riesgo aproximado de $10 \%$.
Espontánea. La perforación espontánea se refiere a la perforación en ausencia de instrumentalización o de trauma externo. La mayoría de los casos corresponde al síndrome de Boerhaave, que ocurre luego de vómitos forzados y arcadas. El mecanismo es un barotrauma dado por un aumento rápido de la presión intraabdominal (que puede llegar a los $200 \mathrm{mmHg}$ ), que en ausencia de relajo del esfínter esofágico superior, se transmite al esófago torácico ${ }^{2,21}$.

Trauma. Se puede categorizar como trauma cerrado o penetrante. El trauma cerrado es muy raro $(0,001 \%)$ y el penetrante básicamente es causado por lesiones cortopunzantes o por armas de fuego y cuenta hasta con $20 \%$ de las causas de perforación esofágica en algunas series ${ }^{2}$.

Cuerpo extraño. La ingestión de cuerpos extraños y la impactación de bolos de comida ocurren frecuentemente, pero la mayoría se resuelve en forma espontánea, inclusive los elementos cortantes y aproximadamente sólo $10-20 \%$ requieren intervención ${ }^{22}$.

Cuando existe lesión por cuerpos extraños se pueden presentar lesiones del tipo perforante y el sitio de perforación ocurre frecuentemente en las zonas de estrechez fisiológica. La lesión puede presentarse en forma espontánea o ser inducida por el endoscopio al tratar de retirar el cuerpo

Tabla 1. Etiología según distintos estudios

\begin{tabular}{|lcrcccccc|}
\hline Estudio & País & $\mathrm{n}$ & Instrumental & Espontánea & Trauma & C extraño & Misceláneas & Quirúrgica \\
\hline Bladergroen 1986 & USA & 127 & $55 \%$ & $15 \%$ & $10 \%$ & $14 \%$ & $6 \%$ & \\
Jones 1992 & USA & 511 & $43 \%$ & $16 \%$ & $19 \%$ & $7 \%$ & $7 \%$ & $8 \%$ \\
Ruiz 1995 & Chile & 16 & $44 \%$ & $31 \%$ & $6 \%$ & $6 \%$ & $14 \%$ & \\
Reeder 1995 & USA & 41 & $70 \%$ & $15 \%$ & $12 \%$ & $0 \%$ & $3 \%$ & \\
Bufkin 1996 & USA & 66 & $73 \%$ & $17 \%$ & $5 \%$ & $0 \%$ & $5 \%$ & \\
Kotsis 1997 & Hungría & 36 & $61 \%$ & $16 \%$ & $5 \%$ & $14 \%$ & $4 \%$ & \\
Mizutani 1997 & Japón & 26 & $42 \%$ & $42 \%$ & $0 \%$ & $16 \%$ & $0 \%$ & \\
Barrientos 1998 & Chile & 18 & $17 \%$ & $28 \%$ & $6 \%$ & $50 \%$ & & \\
Okten 2001 & Turquía & 31 & $80 \%$ & $6,5 \%$ & $6,5 \%$ & $0 \%$ & $6,5 \%$ & \\
Muir 2003 & Irlanda & 75 & $75 \%$ & $17 \%$ & $3 \%$ & $3 \%$ & & $3 \%$ \\
Gupta 2004 & India & 57 & $77 \%$ & $11 \%$ & $4 \%$ & $7 \%$ & $1 \%$ & \\
Hosp Clin U de Chile & Chile & 34 & $32 \%$ & $27 \%$ & & $35 \%$ & $6 \%$ & \\
\hline
\end{tabular}


extraño. Además, puede provocar necrosis por presión y así debilitar la pared, facilitando la lesión por el endoscopio ${ }^{6}$.

Quirúrgica. La perforación en cirugía laparoscópica de tracto digestivo alto es un fenómeno reportado últimamente. Se describen 3 mecanismos que pueden explicar las lesiones en la cirugía de Nissen laparoscópico (que pueden hacerse extensible a los otros procedimientos laparoscópicos altos), como son: la disección inapropiada esofágica posterior, la introducción inadecuada del dilatador o la sonda nasogástrica $\mathrm{y}$, como último, por tracción excesiva de la sutura ${ }^{23}$.

\section{MORBILDAD}

La morbilidad asociada a esta patología es de aproximadamente $40 \%$, siendo las patologías más frecuentes: mediastinitis, neumonía, distress respiratorio y filtraciones persistentes ${ }^{5}$.

\section{MortaLidad}

Es importante tener presente que la etiología, localización y demora en el tratamiento afectan el pronóstico de la enfermedad, siendo el último parámetro el más determinante ${ }^{9,15,24}$. Existen variaciones respecto a la etiología, siendo la de peor pronóstico el síndrome de Boerhaave, describiéndo- se una mortalidad entre 20 y $75 \%{ }^{25}$. Se describe que las perforaciones tratadas posterior a las 24 primeras horas duplicanían la mortalidad ${ }^{16}$. En las Tablas 2, 3, 4 y 5 se describen la relación entre mortalidad y las distintas variables: tiempo, etiología, tipo de tratamiento y localización.

La tasa de mortalidad general en nuestro centro es de $21 \%$, en concordancia con las tasas publicadas, que varían desde 4 a $44 \% 4,5,7,13,15,26,27$. Estudios recientes describen una disminución progresiva de la tasa de mortalidad dado básicamente por un mejor soporte perioperatorio del paciente crítico ${ }^{15}$.

\section{CĹNICA}

La presentación clínica depende de tres factores a mencionar. La localización, el tamaño de la lesión

Tabla 2. M ortalidad según tiempo de diagnóstico

\begin{tabular}{|lccc|}
\hline Autor & Año & $<24 \mathrm{~h}$ & $>24 \mathrm{~h}$ \\
\hline Reeder $^{4}$ & 1995 & $5 \%$ & $14 \%$ \\
Okten $^{13}$ & 2000 & $33 \%$ & $26 \%$ \\
Sung $^{3}$ & 2002 & $0 \%$ & $7 \%$ \\
Muir $^{15}$ & 2003 & $8 \%$ & $44 \%$ \\
Hosp Clin U de Chile & 2004 & $15 \%$ & $28 \%$ \\
\hline
\end{tabular}

Tabla 3. M ortalidad según etiología

\begin{tabular}{|c|c|c|c|c|c|c|}
\hline Autor & Año & Espontánea & Instrumental & Cuerpo ext & Trauma & Quirúrgica \\
\hline Bladergroen $^{8}$ & 1986 & $63 \%$ & $20 \%$ & $6 \%$ & $0 \%$ & \\
\hline Reeder $^{4}$ & 1995 & $20 \%$ & $9 \%$ & & $0 \%$ & \\
\hline Bufkin ${ }^{11}$ & 1996 & $25 \%$ & $25 \%$ & - & $25 \%$ & - \\
\hline Schauer22 & 1996 & & & & & $6 \%$ \\
\hline Lemke $^{20}$ & 1999 & $64 \%$ & & & & \\
\hline Okten ${ }^{13}$ & 2001 & $50 \%$ & $24 \%$ & - & $50 \%$ & - \\
\hline Muir ${ }^{15}$ & 2003 & $15 \%$ & $14 \%$ & $0 \%$ & $0 \%$ & $100 \%$ \\
\hline Kollmar24 & 2003 & $35 \%$ & & & & \\
\hline Gupta 16 & 2004 & $66 \%$ & $5 \%$ & $25 \%$ & $0 \%$ & $33 \%$ \\
\hline Hosp Clin U de Chile & 2004 & $44 \%$ & $9 \%$ & $0 \%$ & & \\
\hline
\end{tabular}


Tabla 4. M ortalidad según manejo

\begin{tabular}{|c|c|c|c|c|c|c|}
\hline Autor & Año & Esofagorrafia & Esofagectomía & Derivación & Conservador & Drenaje \\
\hline Bladergroen $^{8}$ & 1986 & $20 \%$ & $45 \%$ & - & $35 \%$ & $15 \%$ \\
\hline Salo $^{25}$ (mediastinitis) & 1993 & $68 \%$ & $13 \%$ & - & - & - \\
\hline Reeder ${ }^{4}$ & 1995 & $8 \%$ & $14 \%$ & - & $0 \%$ & $0 \%$ \\
\hline Bufkin11 & 1996 & $18 \%$ & $43 \%$ & $0 \%$ & $0 \%$ & $12 \%$ \\
\hline Altorjay 30 & 1997 & & & & $10 \%$ & \\
\hline Altorjay 26 & 1998 & & $3,7 \%$ & & & \\
\hline Okten 13 & 2001 & $16 \%$ & $25 \%$ & $45 \%$ & & - \\
\hline Muir ${ }^{15}$ & 2003 & $8 \%$ & $13 \%$ & - & $20 \%$ & $17 \%$ \\
\hline Gupta $^{16}$ & 2004 & $0 \%$ & $9 \%$ & & $25 \%$ & $25 \%$ \\
\hline Hosp. Clin U de Chile & 2004 & $0 \%$ & $33 \%$ & $36 \%$ & $12 \%$ & - \\
\hline
\end{tabular}

Tabla 5. M ortalidad según localización

\begin{tabular}{|lcccr|}
\hline Autor & Año & Cervical & Torácica & Abdominal \\
\hline Bladergroen $^{8}$ & 1986 & $10 \%$ & $47 \%$ & $59 \%$ \\
Okten $^{13}$ & 2001 & $20 \%$ & $36 \%$ & $0 \%$ \\
Whyte $^{31}$ & 1995 & & $4,5 \%$ & \\
Port5 $^{15}$ & 2003 & & $3,8 \%$ & \\
Muirs $^{15}$ Hosp. Clin U de Chile & 2003 & $9 \%$ & $17 \%$ & $33 \%$ \\
\hline
\end{tabular}

(en relación con el contenido filtrado a los tejidos circulantes) y el tiempo de evolución (grado de respuesta inflamatoria y sepsis). La tríada clásica es dolor, fiebre y presencia de aire subcutáneo o mediastínico. El dolor es el síntoma más frecuente, presente en $70-90 \%$ de los pacientes, usualmente relacionado directamente con el sitio de la perforación ${ }^{1,2}$.

Pacientes con perforaciones cervicales se quejan de dolor cervical, disfagia y odinofagia 2,6 . El cuello está doloroso a la palpación y generalmente se encuentra aire subcutáneo, que en $60 \%$ se detecta por palpación y $95 \%$ por radiología 6 .

En el caso de perforaciones torácicas se presentan como dolor epigástrico, retroesternal 0 lateralizado al lado de la perforación. Las complicaciones respiratorias son comunes siendo el derrame pleural, lo más frecuente, pudiendo provocar disnea y empiema pleural. Se desarrolla sepsis en forma temprana, que si no se trata rápidamente evoluciona a falla multiongánica.

Se describe la tríada de Mackler para el diagnóstico de rotura espontánea, que consiste en dolor torácico, vómitos y enfisema subcutáneo, pero sólo se presenta en aproximadamente $50 \%$ de los casos, por lo que su ausencia no descarta el diagnóstico ${ }^{2}$.

En las perforaciones abdominales, los pacientes presentan epigastralgia que se irradia al dorso $\mathrm{y}$ al hombro izquierdo, junto con signos de irritación peritoneal.

Finalmente, se debe tener en mente que las formas de presentación pueden ser muy variadas y los hallazgos muy sutiles. Por ello, un alto índice de sospecha es crucial para evitar las devastadoras consecuencias de diagnosticar una perforación esofágica en forma tardía. 


\section{Diagnóstico}

Lo más importante para el diagnóstico es plantearlo en forma temprana. Aproximadamente $50 \%$ de los casos tiene una historia atípica e inclusive en un pequeño porcentaje son asintomáticos. Como la primera etiología son los procedimientos endoscópicos, todos los pacientes que presenten dolor, fiebre o algún síntoma inusual luego de una endoscopia alta, especialmente si se realizó algún procedimiento terapéutico, son sospechosos de tener una perforación.

El estudio radiológico es la clave para el diagnóstico. Independiente de la etiología, se deben obtener radiografías de tórax en forma urgente. Se puede realizar el diagnóstico si se encuentra enfisema subcutáneo, neumomediastino o niveles hidroaéreos mediastínicos. También puede sugerir el diagnóstico un ensanchamiento mediastínico, pneumotórax, derrame pleural o infiltrados pulmonares. Hay que tener presente que el estudio radiográfico puede ser negativo si se realizan en forma muy precoz, ya que para que aparezca enfisema mediastínico se estima que tiene que transcurrir aproximadamente una hora de evolución. Al menos 10\% de las radiografías de tórax son normales ${ }^{2}$.

Estudios radiológicos con contraste se realizan para confirmar el diagnóstico y para evidenciar el sitio exacto de perforación. Se recomienda realizar estudio con bario diluido, ya que éste delimita mejor la mucosa esofágica, siendo más sensible para la detección de pequeñas filtraciones. Se reporta que entre 25 y $50 \%$ de las perforaciones no demostradas con agentes hidrosolubles, pueden ser demostradas con bario ${ }^{28}$. Este método presenta hasta $10 \%$ de falsos negativos, que se minimiza si se realiza el estudio con el paciente en decúbito lateral derecho, ya que así se enlentece el paso del medio de contraste, evidenciándose las filtraciones más pequeñas.

La tomografía axial computada (TAC) del tórax puede ser útil en los pacientes en que el estudio inicial es negativo. Realiza el diagnóstico si muestra aire en el mediastino, abscesos adyacentes al esófago o una comunicación entre el esófago y el mediastino.

Cuando una perforación se sospecha en un procedimiento endoscópico se debe explorar el esófago sin insuflar aire, ya que con la presión positiva puede aumentar el paso de contenido por la perforación, convirtiendo eventualmente una perforación pequeña de tratamiento médico a una mayor, requiriendo cirugía ${ }^{29}$. Por esto mismo, no se recomienda a la endoscopia como un elemento primario de diagnóstico ${ }^{1}$.

No debe dudarse en repetir el estudio radiológico si existe una alta sospecha clínica y el estudio inicial es negativo, ya que se describe que el edema de la perforación podría negativizar en forma inicial estos estudios ${ }^{29}$.

\section{MANEjO}

El tratamiento de las perforaciones esofágicas aún es controversial, básicamente porque no existen trabajos que definan una superioridad de un tratamiento sobre otro ${ }^{30}$. Independiente de la modalidad del tratamiento, los objetivos son: prevenir y detener la filtración, eliminar y controlar la infección, mantener el estado nutricional del paciente y restaurar la integridad y continuidad del tracto digestivo.

Manejo no instrumental. Existe éxito con el tratamiento médico de la perforación esofágica, pero requiere una indicación juiciosa. La dificultad reside en predecir qué perforaciones se mantendrán contenidas y cuáles progresarán, con la subsecuente infección no controlada ${ }^{1}$. Es importante la evaluación precoz por un cirujano y que autorice la terapia no-quirúrgica, sobre todo en los casos de perforaciones por endoscopia, ya que así se protege la pérdida de tiempo que puede provocar la tendencia natural del endoscopista que causó la perforación al preferir el manejo conservador ${ }^{29}$. Quedan totalmente excluidos del manejo no quirúrgico los pacientes que presentan perforación libre al espacio pleural o al abdomen, los que tienen neumotórax, los pacientes con falla respiratoria y que presenten obstrucción distal a la perforación de cualquier etiología ${ }^{1,5}$.

Los criterios para el manejo conservador fueron propuestos inicialmente por Cameron en 1979, siendo actualizados por Altorjay en $1997^{31}$ e incluyen:

1. Perforaciones intramurales. 2. Perforación detectada en forma precoz, o si es en forma tardía, que esté circunscrita. 3. Perforación transmural 
que no está ubicada en el abdomen, que está contenida en el mediastino y que drena sin alteraciones al esófago. 4. Perforación que no está asociada con obstrucción esofágica o cáncer. 5 . Síntomas mínimos y sin evidencia clínica de sepsis. 6. Contar con exámenes radiológicos en forma expedita (radiología contrastada y TAC). 7. Contar con el apoyo de un cirujano especialista con experiencia en el tema.

Existe evidencia en la literatura para tratar en forma conservadora a los pacientes que presentan perforación instrumental, especialmente en la región cervical, pequeñas perforaciones que ocurren al dilatar el esófago (cuando la fibrosis periesofágica acompañante bloquea la mediastinitis) y perforaciones de larga evolución (días) con síntomas mínimos donde la indicación no quirúrgica está dada por el organismo en $\mathrm{si}^{29}$.

$\mathrm{Si}$ se cumplen estos criterios, se estaría en condiciones de tratar al paciente con restricción completa de aportes por vía oral, nutrición parenteral total, antibióticos de amplio espectro (cubriendo aerobios y anaerobios) y bloqueadores de la secreción ácida. Se reinicia el aporte oral entre y 7 y 10 días, dependiendo de los resultados de los estudios de contraste de control que se realizan. Es importante destacar que estos pacientes requieren repetidas y continuas evaluaciones para confirmar que se cumplen los criterios para mantener la terapia conservadora, y que cualquier cambio en el cuadro clínico, (especialmente la aparición de signos de sepsis) o la falta de mejoría del paciente exigen el manejo quirúrgico.

En el caso de las perforaciones endoscópicas se recomienda que si son detectadas en forma inmediata, tratar en forma endoscópica con clip o sellante de fibrina. Posterior a esto, realizar control con imágenes y según lo encontrado seguir con tratamiento médico o quirúrgico ${ }^{18}$.

Manejo quirúrgico. Los pilares en las decisiones quirúrgicas se basan en: la localización de la lesión, la presencia de patología esofágica previa, la magnitud de la contaminación, la viabilidad del esófago y el estado general del paciente. La localización es importante ya que las localizaciones cervicales generalmente con un drenaje del espacio paracervical es suficiente ${ }^{1,6}$. La localización de la perforación en el segmento tóracoabdominal se asocia a mayor riesgo de sepsis, por lo cual generalmente requieren resolución quirúrgica 6 .

La presencia de patología esofágica previa orienta a realizar una resección esofágica con reconstrucción inmediata, si las condiciones así lo permiten, o reconstrucción posterior. Esto está dado ya que tiene mayor posibilidad de fallar la reparación primaria ${ }^{1,3}$.

Si existe gran inflamación, contaminación, destrucción de tejido o inestabilidad hemodinámica (todos estos factores en general asociados a presentaciones tardías), la reparación primaria es muy difícil de llevar a cabo y con una alta probabilidad de fracaso, por lo cual, en estas situaciones se recomienda realizar una esofagectomía $1,2,11$.

Existe consenso en que los resultados más favorables se obtienen con la reparación primaria de la perforación, sobre todo en las primeras 24 horas. Reportes recientes muestran un incremento en la sobrevida de los pacientes con perforación en esófagos, sin malignidad, tratados con reparación primaria después de las primeras 24 horas. Con esto se apoya el concepto que siempre debe intentarse el cierre primario, independiente del tiempo de evolución ${ }^{3,5,11,13,32}$. Esta idea fue inicialmente propuesta por Grillo y Wilkins en 1975 y ratificado en 1995 por Whyte y Orringer ${ }^{32}$.

Las lesiones torácicas del tercio superior se acceden por toracotomía derecha y las lesiones del tercio inferior por toracotomía izquierda. Las lesiones de la unión gastroesofágica pueden accederse por toracotomía izquierda o por laparotomía media ${ }^{1}$. En el momento de acceder a la lesión es cuando se debe evaluar cuál es el grado de contaminación, inflamación y destrucción del tejido esofágico para intentar una reparación primaria o para realizar una esofagectomía.

Todo el tejido mediastínico y esofágico desvitalizado debe ser retirado siempre y sólo mucosa, submucosa y muscular sana deben ser anastomosadas $^{1}$. El refuerzo del cierre primario con tejido perilesional viable es controversial. Los que están a favor de esta técnica, argumentan que puede cambiar el curso a una potencial fístula, modificando así la morbi-mortalidad ${ }^{1,3,11}$. Los tejidos para refuerzo que se describen en la literatura incluyen: parche de pericardio, pedículo pleural vascularizado, diafragma, músculo intercostal, músculo extratorácico (romboideo, esternocleido- 
mastoideo, pectoral mayor), fondo gástrico y omento $3,7,33$. Los detractores al refuerzo opinan que es más importante realizar una técnica meticulosa de cierre en dos planos y la eliminación de la obstrucción distal 32 .

En el caso de una lesión esofágica extensa con necrosis transmural o en presencia de una patología esofágica previa (megaesófago, carcinoma, lesión por ingestión de cáusticos, estenosis significativa imposible de dilatar y acalasia grado III-IV), se recomienda realizar una esofagectomía ${ }^{1}$. Esto está bien documentado, básicamente por Salo et al, que reportan una mortalidad de $13 \%$ en los casos manejados con resección en contra de $68 \%$ en casos manejados con tratamiento convencional $^{26}$. En el caso de la acalasia, se recomienda

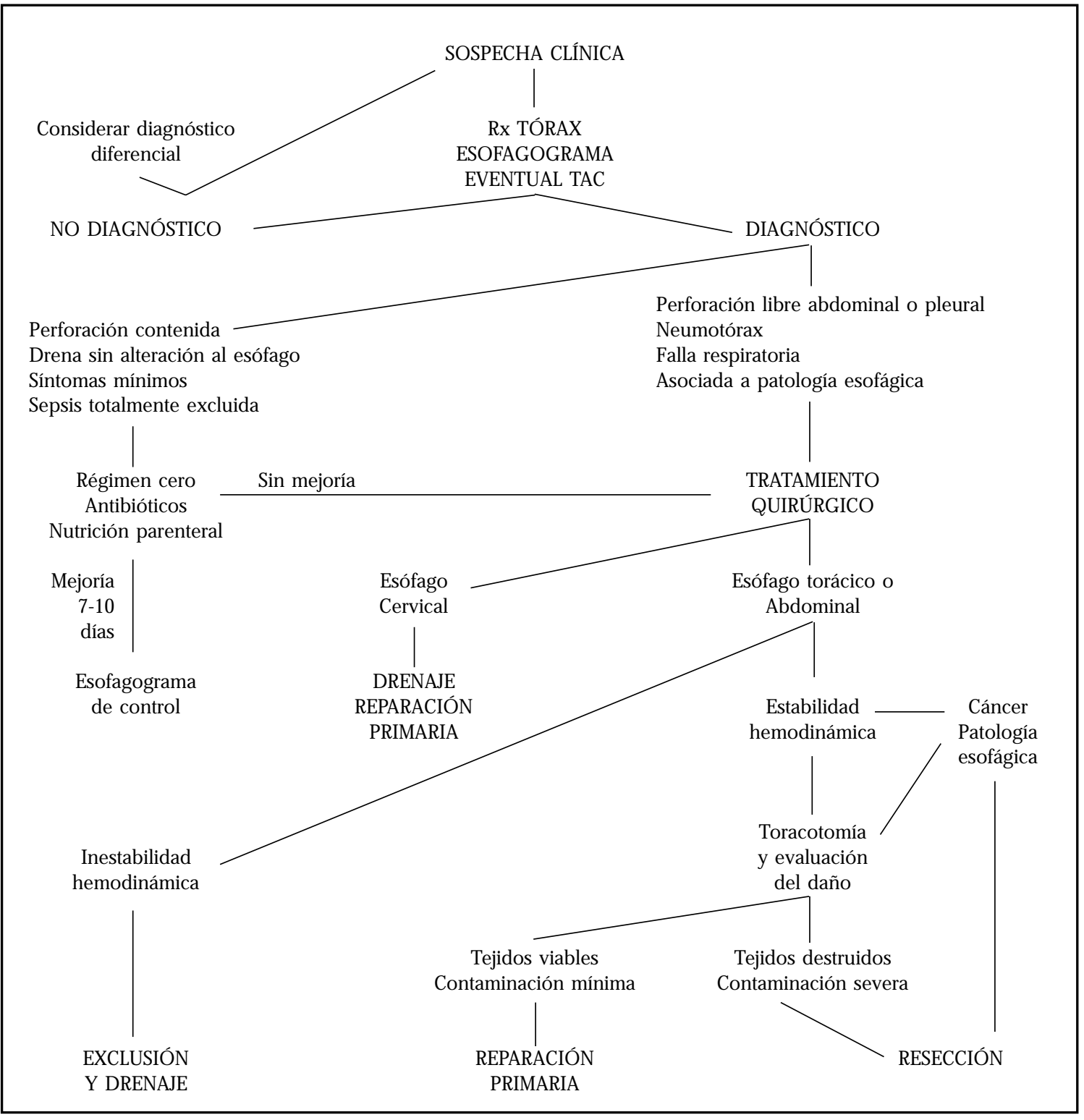

Figura 1. Enfoque clínico de perforación esofágica. 
realizar una reconstrucción con estómago en el mismo tiempo de la esofagectomía, obteniéndose buen resultado ${ }^{17}$. Altorjay et al reportan que no existiría un aumento de la morbilidad operatoria al realizar reconstrucción en un tiempo, aun en casos con sepsis establecida ${ }^{27}$.

En los casos extremos de inestabilidad hemodinámica se recomienda realizar una exclusión y desviación esofágica, que consiste en esofagostoma cervical (fístula salival), cierre del esófago distal y gastrostomía de descompresión gástrica junto con una jejunostomía de alimentación. Existen grupos que no avalan la realización de la exclusión, básicamente porque postulan que el estasis y crecimiento bacteriano en el segmento esofágico excluido podría llevar a una continua inflamación mediastínica y sepsis, con mortalidades de aproximadamente $40 \% 5$. Una alternativa a la exclusión y desviación es la colocación de un tubo $\mathrm{T}$ de drenaje, con lo que se consigue la creación de una fístula esófago-cutánea controlada. Este procedimiento no se recomienda de

\section{REFERENCIAS}

1. Zwischenberger JB, Savage C, Bidani A. Surgical aspects of esophageal disease: perforation and caustic injury. Am J Respir Crit Care Med 2002; 165: 1037-40.

2. Bцом D, Peters J. Esophageal perforation. In: Cameron JL, ed. Current Surgical Therapy. St Louis, MO: Mosby, 2001: 7-12.

3. Sung SW, PARK JJ, KIM YT, KIM JH. Surgery in thoracic esophageal perforation: primary repair is feasible. Dis Esophagus 2002; 15: 204-9.

4. ReEder LB, DeFildpi VJ, Ferguson MK. Current results of therapy for esophageal perforation. Am J Surg 1995; 169: 615-7.

5. Port J, Kent M, Korst R, Bacchetta M, Altorki N. Thoracic Esophageal Perforations: A Decade of Experience. Ann Thorac Surg 2003; 75: 1071-4.

6. Younes Z, Johnson DA. The spectrum of spontaneous and iatrogenic esophageal injury: perforations, Mallory-Weiss tears, and hematomas. J Clin Gastroenterol 1999; 29: 306-17.

7. Kotsis L, Kostic S, Zubovits K. Multimodality treatment of esophageal disruptions. Chest 1997; 112: 1304-9. rutina ya que igualmente puede persistir la filtración con el desarrollo de sepsis, por lo cual estaría indicado en casos de muy alto riesgo ${ }^{1,34}$.

En el caso de perforación en procedimientos laparoscópicos altos y que se reconocen en forma intraoperatoria se pueden reparar en forma exitosa por cierre primario con refuerzo. Schauer et al reportan $0 \%$ de filtraciones postoperatorias en pacientes que sufrieron una perforación laparoscópica y que se repararon en el primer procedimiento. Esto contrasta con $17 \%$ de mortalidad que presentan los pacientes con diagnóstico tardío ${ }^{22}$.

En el caso de cánceres irresecables existen reportes que validan el uso de stent intraluminales como medida de tratamiento con buenos resulta$\operatorname{dos}^{35}$. Igualmente existen reportes recientes en que validan la utilización de stent flexibles cubiertos para el manejo de las perforaciones esofágicas fuera de un contexto maligno ${ }^{33,36}$.

Para finalizar nosotros proponemos el siguiente algoritmo de enfrentamiento de pacientes que presenten esta patología (Figura 1).

8. Bladergroen MR, Lowe Je, Postlethwait RW. Diagnosis and recommended management of esophageal perforation and rupture. Ann Thorac Surg 1986; 42: 235-9.

9. Jones GW, GinSBERG R. Esophageal perforation: a continuing challenge. Ann Thorac Surg 1992; 53: 534

10. RuIz F ET AL. Perforaciones esofágicas. Rev Chil Cir 1995; 47: 56-60.

11. Bufkin BL, Milier JI Jr, Mansour KA. Esophageal perforation: emphasis on management. Ann Thorac Surg 1996; 61: 1447-51.

12. Mizutani $K$, MakuUchi $H$, Tajima $T$, Mitomi T. The diagnosis and treatment of esophageal perforations resulting from nonmalignant causes. Surg Today 1997; 27: 793-800.

13. Okten I, Cangir AK, Ozdemir N, Kavukcu S, Akay H, YAVUZER S. Management of esophageal perforation. Surg Today 2001; 31: 36-9.

14. BarRientos F, Baquerizo A, Muñoz W. Perforación esofágica. Rev Chil Cir 1998; 50: 509-12.

15. Muir A, White J, McGuigan JA, McManus KG, GRAHAM AN. Treatment and outcomes of oesophageal perforation in a tertiary referral center. Eur J Cardio Thorac Surg 2003; 23: 799-804. 
16. Gupta NM, Kaman L. Personal management of 57 consecutive patients with esophageal perforation. Am J Surg 2004; 187: 58-63.

17. Rodríguez A, Braghetto I, Csendes A, Díaz JC, Korn O, Burdiles P, Maluenda F. Resultados Actuales del Manejo de la Perforación Esofágica» Rev Chil Cir 2004; 56: 539-44.

18. Fernández FF, Richter A, Freudenberg S, Wendd $\mathrm{K}$, Manegold BC. Treatment of endoscopic esophageal perforation. Surg Endosc 1999; 13: 962-6.

19. Hernández L, Jacobson J, HaRRis M. Comparison among the perforation rates of Maloney, balloon, and Savary dilatation of esophageal strictures. Gastrointestinal Endoscopy 2000; 51: 60-65.

20. EnNs R, BRanch M. Management of esophageal perforation after therapeutic upper gastrointestinal endoscopy. Gastrointestinal Endoscopy 1998; 47: 318-20.

21. LemKe T, Jagminas L. Spontaneous esophageal rupture: a frequently missed diagnosis. Am Surg 1999; 65: 449-52.

22. EISEN G ET AL. Guideline for the management of ingested foreign bodies. Gastrointestinal Endoscopy 2002; 55: 317-22.

23. Schauer PR, Meyers WC, Eubanks S, Norem RF, FRANKLIN M, PAPPAS TN. Mechanisms of gastric and esophageal perforations during laparoscopic Nissen fundoplication. Ann Surg 1996; 223: 43-52.

24. Gouge TH, Depan HJ, Spencer FC. Experience with the Grillo pleural wrap procedure in 18 patients with perforation of the thoracic esophagus. Ann Surg 1989; 209: 612-17.

25. Kolmar O, Lindemann W, Richter S, Stefren I, Pistorius G, SchiLing MK. Boerhaave's Syndrome: Primary Repair vs. Esophageal Resection-Case Reports and Meta-Analysis of the Literature. J Gastrointest Surg 2003; 7: 726-34.
26. Salo Ja, isolauri Jo, Heikkila lJ, Markkule HT, Heikriman LO, KivilaAkso FO, Mattila SO. Management of delayed esophageal perforation with mediastinal sepsis; esophagectomy or primary repair?J Thor Cardiovasc Surg 1993; 106: 1088-91.

27. Altorjay A, Kiss J, Voros A Sziranyi. The role of esophagectomy in the management of esophageal perforations. Ann Thorac Surg 1998; 65: 1433-6.

28. RuBEsIN, SE, Levine MS. Radiologic diagnosis of gastrointestinal perforation. Radiol Clin N Am 2003; 41: 1095-115.

29. Sawyer R, Phimps C, Vakil N. Short and long term outcome of esophageal perforation. Gastrointestinal Endoscopy 1995; 41: 130-4.

30. Dicks JR, Majeed AW, Stoddard CJ. Omental wrapping of perforated esophagus. Dis Esophagus 1998; 11: 276-8.

31. Altorjay A, Kiss J, Voros A, Bohak A. Nonoperative management of esophageal perforations. Is it justified? Ann Surg 1997; 225: 415-21.

32. Whyte RI, IANNETTONI MD, ORRINGer MB. Intrathoracic esophageal perforation. The merit of primary repair. J Thorac Cardiovasc Surg 1995; 109: 140-4.

33. Mumtaz H, Barone GW, Ketel BL, Ozdemir A. Successful management of a nonmalignant esophageal perforation with a coated stent. Ann Thorac Surg. 2002; 74: 1233-5.

34. OjIma H, Kuwano H, Sasaki S, Fujisawa T, Ishibashi Y. Succesful late management of spontaneous esophageal rupture using $\mathrm{T}$ tube mediastinoabdominal drainage. Am J Surg 2001; 182: 192-6.

35. White R, Mungatana C, Topazian M. Expandable Stents for Iatrogenic Perforation of Esophageal Malignancies. J Gastrointest Surg 2003; 7: 715-20.

36. Siersema PD, Homs M, Haringsma J, Tilanus $\mathrm{H}$, KUIPERS E. Use of large-diameter metallic stents to seal traumatic nonmalignant perforations of the esophagus. Gastrointest Endosc 2003; 58: 356-61. 